\title{
Sonographic Surface Localization of Subcutaneous Foreign Bodies and Masses
}

\author{
Stephanie A. Creel, BSRT(R), RDMS, RVT, Gandikota Girish, MBBS, David A. Jamadar, MBBS, \\ Yoav Morag, MD, Jon A. Jacobson, MD \\ Department of Radiology, University of Michigan, 1500 East Medical Center Drive, Ann Arbor, MI 48103
}

Received 2 June 2008; accepted 27 October 2008

\begin{abstract}
We report a sonographic technique of skin marking of the projection of nonpalpable subcutaneous foreign bodies and masses using a paperclip. Localization and marking of the overlying skin assists in preoperative planning and further management. () 2009 Wiley Periodicals, Inc. J Clin Ultrasound 37: 158-160, 2009; Published online in Wiley InterScience (www.interscience.wiley.com). DOI: 10.1002/jcu.20546
\end{abstract}

Keywords: ultrasound; foreign body; surface localization

$\mathrm{O}$ ccasionally, a small palpable abnormality may be difficult to localize with sonography, particularly if the abnormality is smaller than the thickness of the transducer. Foreign bodies may migrate from their entry site ${ }^{1}$ and similarly may be difficult to localize, particularly if small in size. Accurate surface localization of masses and foreign bodies is important before removal. ${ }^{2}$ We describe a simple sonographic technique to mark the projection on the skin of small masses or foreign bodies in the subcutaneous tissue.

\section{ULTRASOUND TECHNIQUE}

We use an HDI 5000 scanner and 10-15 MHz linear-array transducer (Philips ATL, Bothell, WA). A large amount of acoustic coupling gel is used. A paperclip (Jumbo nonskid paperclips, OM99146, OfficeMax North America, Inc. Itasca, IL) is also used to assist in marking the skin overlying the subcutaneous abnormality.

Correspondence to: G. Girish

(C) 2009 Wiley Periodicals, Inc.
Opening a segment of a paperclip allows the linear segment to be placed between the transducer and the skin, with the coiled part used for stability. The paperclip produces a hyperechoic comet tail artifact. The paperclip itself produces a bright echo that is usually well seen on the focally depressed skin surface (Figure 1B). ${ }^{3,4,5,6}$ The comet tail artifact produced by the paperclip serves as a marker through the imaged soft tissues. By moving the paperclip under the probe, the comet tail artifact can be moved directly over the area of interest, with the clip localizing it on the skin surface.

\section{Surface Marking of a Linear Foreign Body}

The transducer is aligned along the long axis of the foreign body (Figure 1A). The paperclip is adjusted to lie at 90 degrees to the long axis of the transducer. The comet tail artifact created by the paperclip can be visualized as the paperclip is moved on the skin surface under the stationary probe facilitating precise localization of the artifact over 1 end of the foreign body (Figure 1A, B). The clip is held in this position, the transducer removed, and acoustic jelly wiped away, at which time an ink marker is used to draw a line over the straight end of the paperclip (Figure 1A; line A). This process is repeated with the paperclip placed at the other end of the foreign body (Figure 1A, C; line B). Finally, the clip is placed parallel to the probe in such a way that the artifact overlies the foreign body and a line is drawn as before (line $\mathrm{C}$ ). On the skin, an "H"-shaped mark is produced where the transverse arm of "H" overlies the foreign body. 


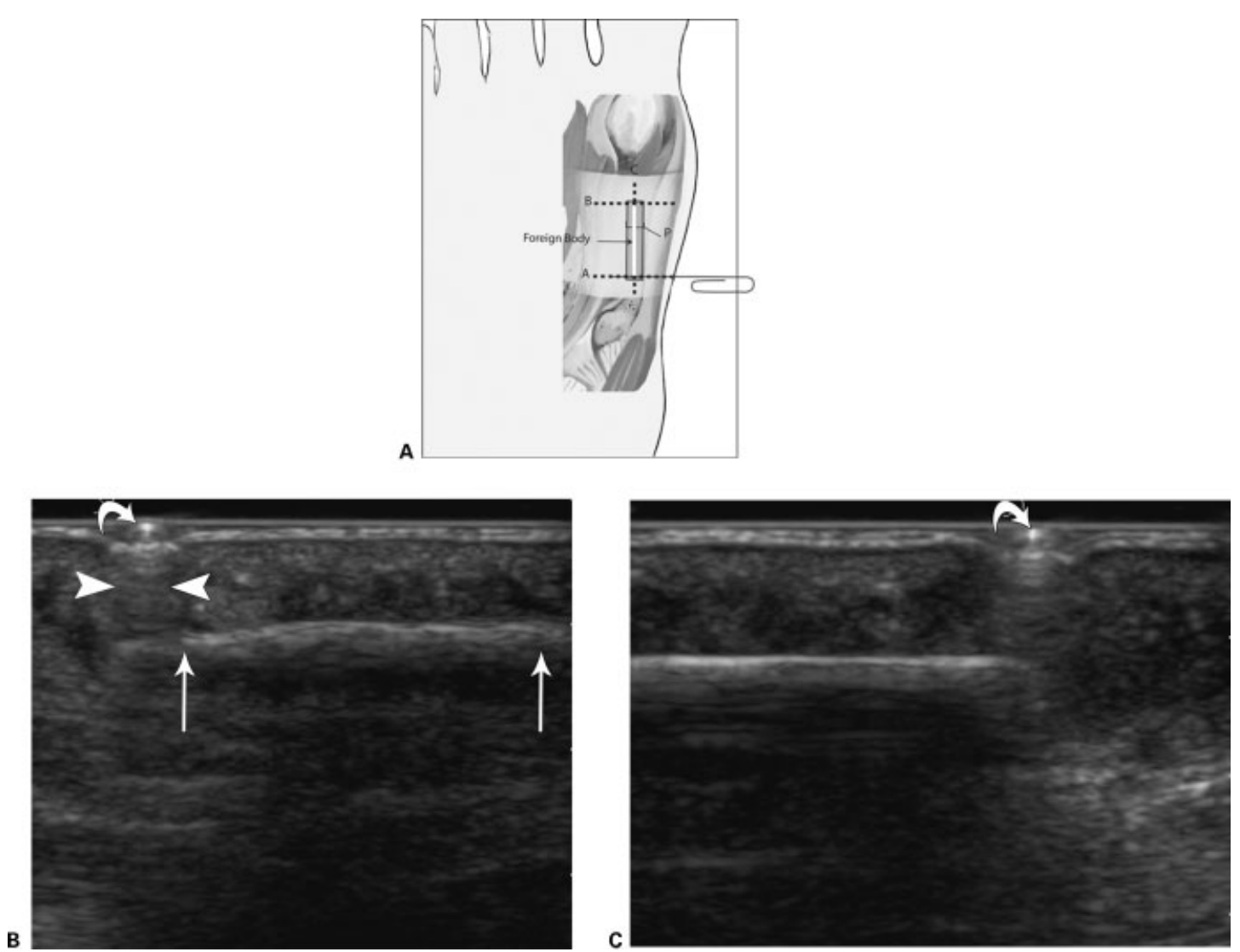

FIGURE 1. Diagram shows the relative positions of the sonographic transducer and paperclip over a linear foreign body in the soft tissues on the plantar aspect of the foot. (A) The transducer $(\mathrm{P})$ is placed longitudinal to the underlying foreign body. The open end of the paperclip is moved to position A transverse to the foreign body to localize the proximal end of the foreign body, and line A is drawn on the skin along the paperclip. B: The paperclip is then moved distally and the process repeated with line B drawn at the distal end of the foreign body. C: The paperclip is then placed over and parallel to the foreign body and line $C$ is drawn. Line $C$ between line $B$ and line $A$ shows the length and location of the underlying linear foreign body. (B) A 42-year-old woman with a toothpick in the soft tissues at the plantar aspect of the left foot. The paperclip (curved arrow) is placed over the proximal end of the foreign body (arrows), (corresponding to position A in Figure 1A). Note the comet tail artifact (arrow heads) from the paperclip and mixed shadowing and reverberation artifact deep to the toothpick. (C) The paperclip (curved arrow) is repositioned so the comet tail artifact of the paperclip coincides with the distal end of the foreign body (corresponding to position B in Figure 1A).

\section{Localization of a Nonpalpable Mass}

The transducer is placed along the long axis of the mass (Figure 2A; probe P2), and the paperclip is placed transversely to the long axis of the transducer between the transducer and the skin. The comet tail artifact is then moved until it is directly over the mass (Figure 2B). A line is drawn alongside the paperclip on the skin (Figure 2A; line B). The transducer is rotated 90 degrees to its original position, the paperclip is repositioned perpendicularly to the long axis of the transducer and moved over the mass, and a second line is placed on the skin alongside the paperclip (Figure 2A; line A). The intersection of the 2 lines on the skin (Figure 2A; point E) localizes the projection of the center of the mass.

\section{DISCUSSION}

Sonography is an effective means for localization of soft-tissue masses and foreign bodies, which minimizes the surgical exploration. Real-time sonography can also be used to guide the percutaneous removal of a soft-tissue foreign body. ${ }^{2}$

The use of the paperclip technique described here provides accurate 2-dimensional surface localization of an underlying structure, mass, or foreign body. In addition, the depth of the abnormality from the skin can be measured on the sonographic image, which provides a 3 -dimensional localization. It is critical that the sonographic transducer always remain perpendicular to the skin surface. Care should be taken to ensure that the patient is positioned for the sonographic examination as the patient would be positioned for surgery. 


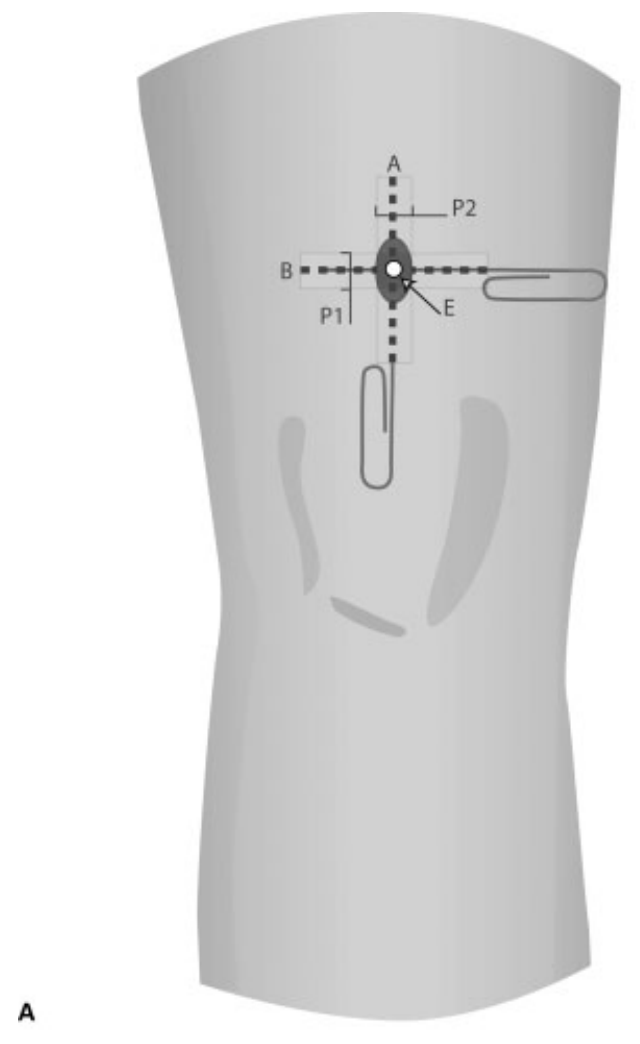

A

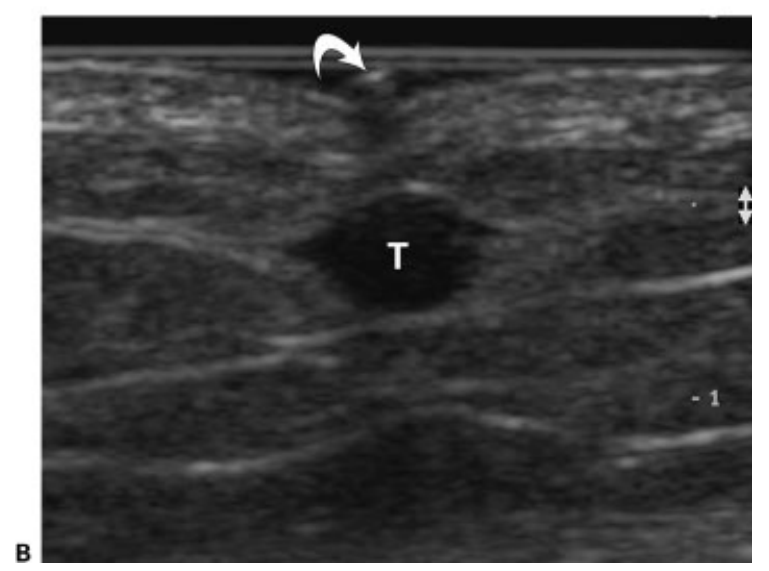

FIGURE 2. (A) Diagram shows the relative positions of the transducer and paperclip over a mass in the soft tissues at the anterior aspect of the distal thigh. A: The transducer (P1) is positioned along the short axis of the mass. The paperclip is placed perpendicularly to the transducer and a line $A$ is drawn over the paperclip. B: The transducer (P2) is repositioned 90 degrees to its initial position, now along the long axis of the mass and the paperclip positioned perpendicular to the transducer along line $B$. The intersection of lines $A$ and $B$ (point $E$ ) marks the center of the ovoid lesion (arrow). $95 \times 199 \mathrm{~mm}(300 \times 300 \mathrm{dpi})$. (B) A 55-year-old woman with malignant melanoma and a nonpalpable subcutaneous metastatic deposit in the distal thigh. The echogenic paperclip (curved arrow) is positioned over a hypoechoic metastatic deposit ( $T$ ). Note a comet tail artifact deep to the paperclip providing a surface landmark. 95 $\times 73 \mathrm{~mm}(300 \times 300 \mathrm{dpi})$.
The paperclip technique can be used for both central localization of a mass as well as the both ends of a linear foreign body.

A nonlinear foreign body may require more creativity. Marking the limits of the long axis dimension and 1 or 2 points along its more central portion with a description of the rough dimensions and shape as well as the depth of the foreign body will usually suffice.

Several nonradiopaque soft tissue foreign bodies, such as wood and plastic, remain undetected at radiography. ${ }^{7-10}$ These foreign bodies are visualized as hyperechoic on sonography. ${ }^{10}$ Sonography can be used effectively to locate wooden foreign bodies as small as $2.5 \mathrm{~mm}$ in length ${ }^{7}$ or 0.5 mm thick. ${ }^{8}$ Similarly, minute subcutaneous masses can be localized on the skin surface before surgical excision.

\section{ACKNOWLEDGMENT}

We thank Anne Phillips for her help with the artwork and formatting the images.

\section{REFERENCES}

1. Fornage BD. Preoperative sonographic localization of a migrated transosseous stabilizing wire in the hand. J Ultrasound Med 1987;6:471.

2. Shiels WE $2^{\text {nd }}$, Babcock DS, Wilson JL, et al. Localization and guided removal of soft-tissue foreign bodies with sonography. AJR Am J Roentgenol 1990;155:1277.

3. Fornage BD, Schernberg FL. Sonographic diagnosis of foreign bodies of the distal extremities. AJR Am J Roentgenol 1986;147:567.

4. Fornage BD, Schernberg FL. Sonographic preoperative localization of a foreign body in the hand. J Ultrasound Med 1987;6:217.

5. Rubin JM, Adler RS, Bude RO, et al. Clean and dirty shadowing at US: a reappraisal. Radiology 1991;181:231.

6. Scanlan KA. Sonographic artifacts and their origins. AJR Am J Roentgenol 1991;156:1267.

7. Jacobson JA, Powell A, Craig JG, et al. Wooden foreign bodies in soft tissue: detection at US. Radiology 1998;206:45.

8. Failla JM, van Holsbeeck M, Vanderschueren G. Detection of a $0.5-\mathrm{mm}$-thick thorn using ultrasound: a case report. J Hand Surg (Am) 1995;20: 456.

9. Felman AH, Fisher MS. The radiographic detection of glass in soft tissue. Radiology 1969;92:1529.

10. Horton LK, Jacobson JA, Powell A, et al. Sonography and radiography of soft-tissue foreign bodies. AJR Am J Roentgenol 2001;176:1155. 